\title{
RESISTÊNCIA DE Conyza canadensis E C. bonariensis AO HERBICIDA GLYPHOSATE $^{1}$
}

\author{
Glyphosate-Resistance in Conyza canadensis and $\boldsymbol{C}$. bonariensis
}

MOREIRA, M.S. ${ }^{2}$, NICOLAI, M. ${ }^{2}$, CARVALHO, S.J.P. ${ }^{2}$ e CHRISTOFFOLETI, P.J. ${ }^{3}$

\begin{abstract}
RESUMO - O objetivo deste trabalho foi avaliar, por meio de curvas de dose-resposta, a ocorrência de biótipos resistentes ao herbicida glyphosate em populações de Conyza canadensis e C. bonariensis, bem como propor tratamentos alternativos para esses biótipos. Os experimentos foram realizados em casa de vegetação, utilizando-se três populações de cada espécie: duas com suspeita de resistência ao herbicida glyphosate, coletadas em pomares de laranja localizados em duas regiões diferentes do Estado de São Paulo; e uma suscetível, coletada em área sem histórico de aplicação do herbicida. O delineamento experimental adotado foi o de blocos ao acaso, com quatro repetições. Para cada espécie, os tratamentos foram resultado da combinação fatorial entre as três populações e os tratamentos herbicidas (oito doses de glyphosate ou cinco tratamentos alternativos). As doses de glyphosate foram (g e.a. ha ${ }^{-1}$ ): 90, 180, 360, 720, 1.440, 2.880, 5.760 e testemunha sem aplicação. Como alternativas de controle, foram testados os seguintes tratamentos $\left(\mathrm{g} \mathrm{ha}^{-1}\right)$ : glyphosate $+2,4-\mathrm{D}$ $(1.440+1.005)$, glyphosate + metsulfuron $(1.440+2,4)$, glyphosate + metsulfuron $(1.440+$ $3,6)$, glyphosate + metribuzin $(1.440+480)$ e testemunha sem aplicação. A partir dos resultados, comprovou-se a existência de populações de ambas as espécies com biótipos resistentes ao herbicida glyphosate, com diferentes níveis de resistência. Todos os tratamentos herbicidas alternativos controlaram de forma eficiente as três populações de cada espécie.
\end{abstract}

Palavras-chave: buva, EPSPs, biótipos, populações, plantas daninhas.

\begin{abstract}
The objective of this research was to evaluate, through dose-response curves, the occurrence of glyphosate-resistant biotypes in populations of Conyza canadensis and C. bonariensis; as well as to propose alternative treatments to these biotypes. The experiments were conducted in a greenhouse, using three populations of each species: two with suspicion of resistance to the herbicide glyphosate, and collected in orange orchards located in two different São Paulo state regions; and a susceptible one, collected in an area without herbicide application history. The experimental design adopted was randomized blocks, with four replicates. For each species, the treatments were the result of a factorial combination among the three populations and the herbicide treatments (eight rates of glyphosate or five alternative treatments). The glyphosate rates used were (g e.a. $\left.\mathrm{ha}^{-1}\right)$ : 90, 180, 360, 720, 1.440, 2.880, 5.760 and checks

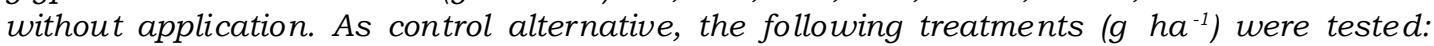
glyphosate $+2,4-D(1.440+1.005)$, glyphosate + metsulfuron $(1.440+2,4)$, glyphosate + metsulfuron $(1.440+3,6)$, glyphosate + metribuzin $(1440+480)$ and checks without application. The results obtained proved the existence of populations of both species with glyphosate-resistant biotypes, with different levels of resistance. All the alternative herbicide treatments controlled efficiently the three populations of each species.
\end{abstract}

Keywords: horseweed, EPSPs, biotypes, populations, weeds.

1 Recebido para publicação em 17.7.2006 e na forma revisada em 27.2.2007.

Parte da dissertação do primeiro autor, para obtenção do título de Mestre em Agronomia pela Escola Superior de Agricultura "Luiz de Queiroz" - ESALQ/USP.

2 Eng.-Agr., Alunos do Programa de Pós-Graduação em Fitotecnia, ESALQ/USP, <murilosm@esalq.usp.br>, $<$ marcelon@esalq.usp.br>, <sjpcarvalho@yahoo.com.br>3,3 Professor Associado do Departamento de Produção Vegetal, ESALQ/ USP, Caixa Postal 09, 13419-900 Piracicaba-SP, <pjchrist@esalq.usp.br>. 


\section{INTRODUÇÃO}

A resistência de plantas daninhas a herbicidas é definida como a capacidade natural e herdável de determinados biótipos, dentro de uma população, de sobreviver e se reproduzir após a exposição a doses de herbicidas que seriam letais a indivíduos normais (suscetiveis) da mesma espécie (Christoffoleti \& LópezOvejero, 2004). A resistência de plantas daninhas aos herbicidas é um fenômeno natural que ocorre espontaneamente em suas populações, não sendo, portanto, o herbicida o agente causador, mas sim selecionador dos indivíduos resistentes que se encontram em baixa freqüência inicial (Christoffoleti et al., 1994).

Atualmente, o glyphosate é um dos herbicidas de maior importância mundial, sendo utilizado por muitos anos no controle de plantas daninhas anuais ou perenes em diversos sistemas de produção (Faircloth et al., 2001; Blackshaw \& Harker, 2002). A mais nova forma de uso do glyphosate na agricultura é a aplicação em culturas geneticamente modificadas para tolerância ao produto, o que também pode contribuir significativamente para seleção de biótipos resistentes em espécies de plantas daninhas (Koger \& Reddy, 2005).

O glyphosate age inibindo a enzima 5-enolpiruvilshiquimato-3-fosfato sintase (EPSPs), que é a responsável pela reação de conversão do shiquimato-3-fosfato e fosfoenolpiruvato em EPSP e fosfato inorgânico, na rota do ácido shiquímico (Geiger \& Fuchs, 2002). A inibição da EPSPs resulta no acúmulo de ácido shiquímico nas plantas e na redução da biossíntese de aminoácidos aromáticos, como triptofano, tirosina e fenilalanina.

Atualmente existem sete espécies de plantas daninhas com casos relatados de biótipos resistentes ao glyphosate: Eleusine indica, na Malásia (Tran et al., 1999; Lee \& Ngim, 2000); Lolium rigidum, na Austrália e nos Estados Unidos (Powles et al., 1998; Pratley et al., 1999; Simarmata et al., 2003); Plantago lanceolata, na África do Sul (Heap, 2004); Conyza bonariensis, na África do Sul e Espanha (Urbano et al., 2005); Lolium multiflorum, no Brasil, Chile e nos Estados Unidos (Perez \& Kogan, 2003; Christoffoleti et al., 2005; PerezJones et al., 2005); Conyza canadensis, nos Estados Unidos e na África do Sul (VanGessel, 2001; Koger et al., 2004; Main et al., 2004); e
Ambrosia artemisifolia, nos Estados Unidos (Sellers et al., 2005).

Com relação a Conyza canadensis (buva), o primeiro relato sobre biótipos resistentes ao glyphosate ocorreu nos Estados Unidos, no estado de Delaware, em 2001 (VanGessel, 2001). Desde então, biótipos de C. canadensis resistentes ao glyphosate foram encontrados em diversos outros estados norte-americanos, como no Tennessee (Mueller et al., 2003), Mis sis sipi (Koger et al., 2004), Kentucky, Indiana, Maryland, New Jersey, Ohio, Arkansas e Carolina do Norte, além de outros países, como a África do Sul (Heap, 2004).

C. canadensis e C. bonariensis (buvas) são espécies originárias dos Estados Unidos (Weaver et al., 2001), pertencentes à família Asteraceae, que possuem ciclo de desenvolvimento anual. São espécies extremamente prolíficas, podendo produzir até 200.000 sementes viáveis por planta, estabelecendo-se em diversas condições climáticas. São plantas que apresentam boa adaptabilidade em sistemas conservacionistas de solo, como: plantio direto, cultivo mínimo e áreas de fruticultura (Bhowmik \& Bekech, 1993). A habilidade de autopolinização da espécie aliada à grande produção de sementes facilmente dispersáveis são fatores que podem contribuir para a boa adaptabilidade ecológica, para a sobrevivência de biótipos resistentes de buva e para as altas infestações nos sistemas conservacionistas de solo.

A intensa utilização de glyphosate nas áreas citrícolas do Estado de São Paulo favorece o aumento da pressão de seleção, que, aliado à boa adaptabilidade ecológica das espécies de buva (C. canadensis e C. bonariensis) a sistemas conservacionistas de manejo de solo, contribui para a seleção de biótipos resistentes dessas espécies. Dessa forma, o objetivo deste trabalho foi avaliar, por meio de curvas de doseresposta, a ocorrência de biótipos resistentes ao herbicida glyphosate em populações brasileiras de $C$. canadensis e $C$. bonariensis, bem como propor tratamentos alternativos para tais biótipos.

\section{MATERIAL E MÉTODOS}

Os experimentos foram instalados em casa de vegetação no Departamento de Produção Vegetal da Escola Superior de Agricultura "Luiz 
de Queiroz" - ESALQ/USP, localizado em Piracicaba-SP, durante os meses de janeiro a março de 2006. No trabalho, utilizaram-se três populações de $C$. canadensis e $C$. bonariensis, duas coletadas em áreas com suspeita de resistência, no Estado de São Paulo (R1 e R2), e uma suscetivel (S), coletada em áreas sem histórico de aplicação de glyphosate, em Piracicaba-SP (Tabela 1).

As sementes foram coletadas quando as plantas se apresentavam em estádio de plena maturidade. Posteriormente, foram devidamente identificadas pela região e pela espécie. A semeadura foi feita em bandejas plásticas preenchidas com substrato comercial e terra arenosa na proporção de 1:1. Inicialmente as bandejas foram identificadas e irrigadas até atingir a máxima capacidade de armazenamento de água. As sementes foram depositadas nas bandejas e cobertas por uma fina camada de terra arenosa. As bandejas foram então cobertas com jornal para minimizar o risco da contaminação de sementes entre elas, visto a grande facilidade de dispersão destas.

Aproximadamente uma semana após a semeadura, grande parte das sementes havia germinado. Quando as plantas apresentavam duas folhas verdadeiras, foi realizado o transplante para vasos com capacidade para $1 \mathrm{~L}$, preenchidos com substrato comercial devidamente adubado, na densidade de uma planta por vaso. Os vasos foram mantidos em casa de vegetação e irrigados diariamente.

Quando as plantas apresentavam cinco folhas verdadeiras, realizou-se a aplicação dos tratamentos herbicidas. Para isso, utilizou-se a câmara de aplicação do Departamento de Produção Vegetal da ESALQ/USP, equipada com bico tipo leque, modelo Teejet 80.02,

Tabela 1 - Espécie, origem, identificação (sigla) e suspeita, referente a todas as populações de Conyza canadensis e C. bonariensis estudadas no trabalho. Piracicaba, 2006

\begin{tabular}{|l|l|l|l|}
\hline \multicolumn{1}{|c|}{ Espécie } & \multicolumn{1}{|c|}{ Origem } & \multicolumn{1}{c|}{ Sigla } & Suspeita \\
\hline Conyza canadensis & Matão - SP & CCR1 & Resistente \\
Conyza canadensis & Cajobi - SP & CCR2 & Resistente \\
Conyza canadensis & Piracicaba - SP & CCS & Suscetível \\
\hline Conyza bonariensis & Matão - SP & CBR1 & Resistente \\
Conyza bonariensis & Cajobi - SP & CBR2 & Resistente \\
Conyza bonariensis & Piracicaba -SP & CBS & Suscetível \\
\hline
\end{tabular}

calibrado na altura de $0,50 \mathrm{~m}$ da superficie do alvo e com consumo relativo de calda da ordem de $200 \mathrm{~L} \mathrm{ha}^{-1}$.

Os tratamentos foram resultado da combinação fatorial entre as três populações de cada espécie e oito doses de glyphosate ou cinco tratamentos alternativos. As doses de glyphosate utilizadas foram (g e.a. ha-1): 90, 180, 360, 720, $1.440,2.880,5.760$ e testemunha sem aplicação. Os tratamentos alternativos for am (g ha $\left.{ }^{-1}\right)$ : glyphosate + metsulfuron $(1.440+2,4$ e $1.440+3,6)$, glyphosate + 2,4-D $(1.440+$ 670), glyphosate + metribuzin $(1.440+480)$ e testemunha sem aplicação de herbicidas. O delineamento experimental foi o de blocos ao acaso, com quatro repetições. Os produtos e doses usados no experimento estão detalhadamente descritos na Tabela 2.

Avaliou-se o controle percentual e a massa seca residual aos 28 dias após a aplicação (DAA). Para isso, foi atribuído $0 \%$ no caso da ausência de sintomas causados pelos herbicidas e $100 \%$ para a morte das plantas. A massa seca foi obtida a partir da colheita do material vegetal remanescente nas parcelas, com posterior secagem em estufa a $70{ }^{\circ} \mathrm{C}$ por 48 horas. A massa seca foi corrigida para valores percentuais por meio da comparação da massa obtida nos tratamentos herbicidas com a massa da testemunha, considerada $100 \%$.

Os dados foram inicialmente submetidos à aplicação do teste F sobre a análise de variância. Os dados do experimento com tratamentos alternativos foram então comparados por meio da aplicação do teste de Tukey a 5\%. Os dados do experimento de curvas de dose-resposta foram ajustados ao modelo de regressão não-linear do tipo logístico. A variável controle foi ajustada ao modelo proposto por Streibig et al. (1988):

$$
y=\frac{a}{\left[1+\left(\frac{x}{b}\right)^{c}\right]}
$$

em que: $y=$ porcentagem de controle; $x=$ dose do herbicida; e $a, b$ e $c=$ parâmetros da curva, de modo que $a$ é a diferença entre o ponto máximo e mínimo da curva, $b$ é a dose que proporciona $50 \%$ de resposta da variável e $c$ é a declividade da curva. 
Tabela 2 - Tratamentos herbicidas aplicados sobre todas as populações de Conyza canadensis e C. bonariensis. Piracicaba, 2006

\begin{tabular}{|c|c|c|c|}
\hline \multicolumn{2}{|c|}{ Herbicida } & \multicolumn{2}{|c|}{ Dose } \\
\hline Nome comum & Nome comercial & $\mathrm{g}^{1 /} \mathrm{ha}^{-1}$ & g ou $\mathrm{mL}$ p.c..$^{2 /} \mathrm{ha}^{-1}$ \\
\hline 01. testemunha & - & - & - \\
\hline \multicolumn{4}{|c|}{ Curva de dose-resposta } \\
\hline 02. glyphosate & Roundup WG & 90 & 125 \\
\hline 03. glyphosate & Roundup WG & 180 & 250 \\
\hline 04. glyphosate & Roundup WG & 360 & 500 \\
\hline 05. glyphosate & Roundup WG & 720 & 1.000 \\
\hline 06. glyphosate & Roundup WG & 1.440 & 2.000 \\
\hline 07. glyphosate & Roundup WG & 2.880 & 4.000 \\
\hline 08. glyphosate & Roundup WG & 5.760 & 8.000 \\
\hline \multicolumn{4}{|c|}{ Herbicida alternativo } \\
\hline 09. glyphosate $+2,4-\mathrm{D}$ & Roundup WG + DMA 806 BR & $1.440+1.005$ & $2.000+1.500$ \\
\hline 10. glyphosate + metsulfuron* & Roundup WG + Ally & $1.440+2,4$ & $2.000+4,0$ \\
\hline 11. glyphosate + metsulfuron* & Roundup WG + Ally & $1.440+3,6$ & $2.000+6,0$ \\
\hline 12. glyphosate + metribuzin & Roundup WG + Sencor 480 & $1.440+480$ & $2.000+1.000$ \\
\hline
\end{tabular}

${ }^{1 /}$ Ingrediente ativo - equivalente ácido ou ingrediente ativo; ${ }^{2}$ produto comercial; * adição de Assist $0,1 \mathrm{v} / \mathrm{v}$.

Para a variável massa seca residual, adotou-se o modelo proposto por Seefeldt et al. (1995):

$$
y=a+\frac{b}{\left[1+\left(\frac{x}{c}\right)^{d}\right]}
$$

em que: $y=$ porcentagem residual da massa seca; $x=$ dose do herbicida; e $a, b, c$ e $d=$ parâmetros da curva, de modo que $a$ é o limite inferior da curva, $b$ é a diferença entre o ponto máximo e mínimo da curva, $c$ é a dose que proporciona $50 \%$ de resposta da variável e $d$ é a declividade da curva.

O modelo logístico apresenta vantagens, uma vez que um dos termos integrantes da equação é uma estimativa do valor de $\mathrm{C}_{50}$ ou de $\mathrm{GR}_{50}$ (Christoffoleti, 2002). $\mathrm{O} \mathrm{C}_{50}$ (control by $50 \%$ ) e o $\mathrm{GR}_{50}$ (growth reduction by 50\%) são as doses do herbicida que proporcionam $50 \%$ de controle ou de redução de massa da planta daninha, respectivamente (Christoffoleti, 2002; Christoffoleti \& López-Ovejero, 2004). Embora um dos parâmetros do modelo logístico seja uma estimativa do valor de $\mathrm{C}_{50}$, optou-se por realizar seu cálculo matemático por meio da equação inversa, conforme discussão proposta por Carvalho et al. (2005).

Com os valores de $\mathrm{C}_{50}$ ou $\mathrm{GR}_{50}$ obteve-se o fator de resistência (F) para cada combinação das populações com suspeita de resistência e a população suscetível de cada espécie. O fator de resistência $(F=R / S)$ expressa o número de vezes em que a dose necessária para controlar $50 \%$ da população resistente é superior à dose que controla $50 \%$ da população suscetivel (Hall et al., 1998; Christoffoleti, 2002).

\section{RESULTADOS E DISCUSSÃO}

\section{Conyza canadensis}

Na Figura 1, observa-se que a dose recomendada de glyphosate $\left(720 \mathrm{~g} \mathrm{ha}^{-1}\right)$ proporcionou controle da ordem de apenas 30\% para as populações com suspeita de resistência e superior a $90 \%$ para a população suscetivel da espécie. Resultados semelhantes foram encontrados por VanGessel (2001), em que o controle do biótipo resistente foi de apenas $50 \%$ na dose de $720 \mathrm{~g} \mathrm{ha}^{-1}$. Esses resultados indicam a condição de resistência das populações provenientes dos pomares de citros do Estado de São Paulo, provavelmente selecionadas pelas aplicações rotineiras do produto. 


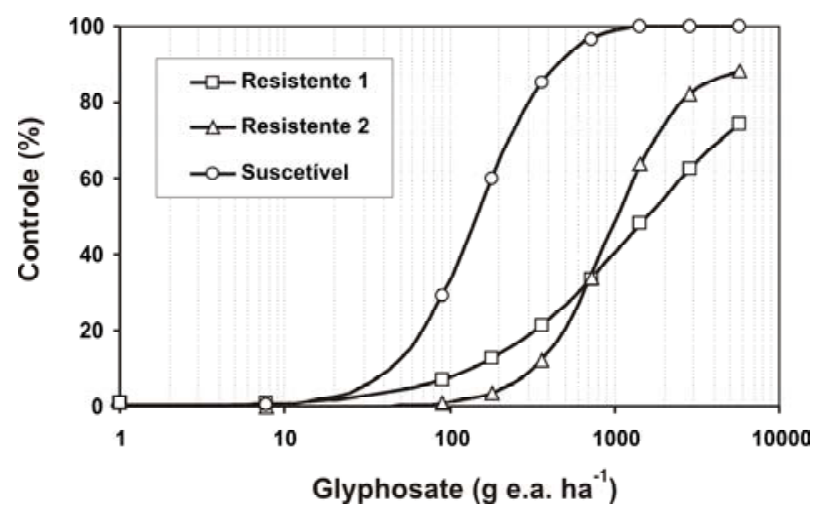

Figura 1 - Controle percentual de três populações de Conyza canadensis, duas supostamente resistentes e uma suscetível, quando submetidas a diferentes doses do herbicida glyphosate, avaliado aos 28 DAA. DMS ${ }_{\text {população }}=12,66$. Piracicaba, 2006.

A variável massa seca residual está em concordância com o que foi discutido anteriormente, em que, novamente, foram necessárias aplicações de doses maiores sobre as populações com suspeita de resistência para obtenção da mesma redução obtida para a população suscetivel (Figura 2). Nesse caso, nem mesmo as doses mais concentradas de glyphosate foram suficientes para controlar as populações resistentes, visto que para a dose de $5.760 \mathrm{~g} \mathrm{ha}^{-1}$ de glyphosate ainda restaram aproximadamente $20 \%$ de massa seca residual (Figura 2).

Com relação aos tratamentos alternativos, todas as combinações testadas alcançaram controle de $100 \%$ de todas as populações, aos

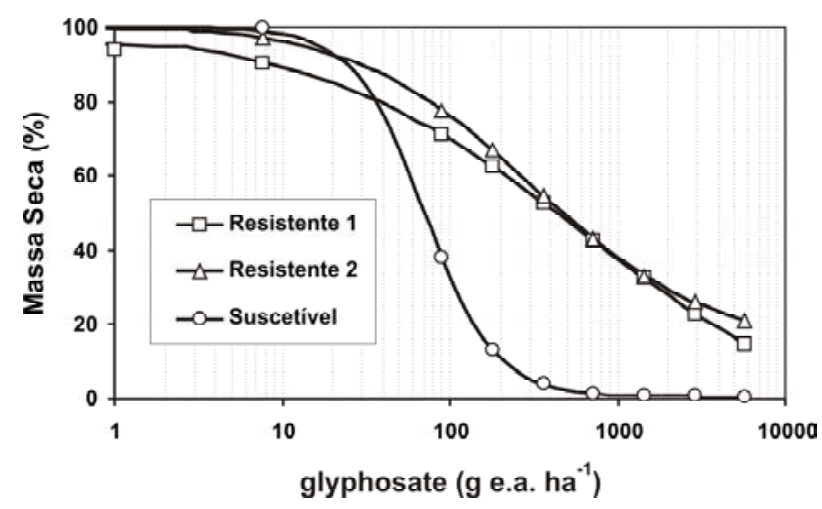

Figura 2 -Massa seca residual (\%) de três populações de Conyza canadensis, duas supostamente resistentes e uma suscetível, quando submetidas a diferentes doses do herbicida glyphosate, avaliada aos 28 DAA. DMS população $=22,3$. Piracicaba, 2006.
28 DAA. Assim, considera-se que o uso conjunto de glyphosate com 2,4-D, metsulfuron ou metribuzin é alternativa viável para o controle de populações de $C$. canadensis resistentes ao herbicida glyphosate (dados não apresentados).

\section{Conyza bonariensis}

Os resultados obtidos para as populações de $C$. bonariensis são bastante semelhantes aos verificados para C. canadensis, demonstrando também a existência de populações dessa espécie resistentes ao glyphosate. Na Figura 3, pode-se verificar que a dose recomendada do produto $\left(720 \mathrm{~g} \mathrm{ha}^{-1}\right)$ controlou adequadamente a população suscetível de $C$. bonariensis, enquanto para as populações supostamente resistentes foram necessárias doses da ordem de $5.760 \mathrm{~g} \mathrm{ha}^{-1}$ para obtenção de cerca de $80 \%$ de controle.

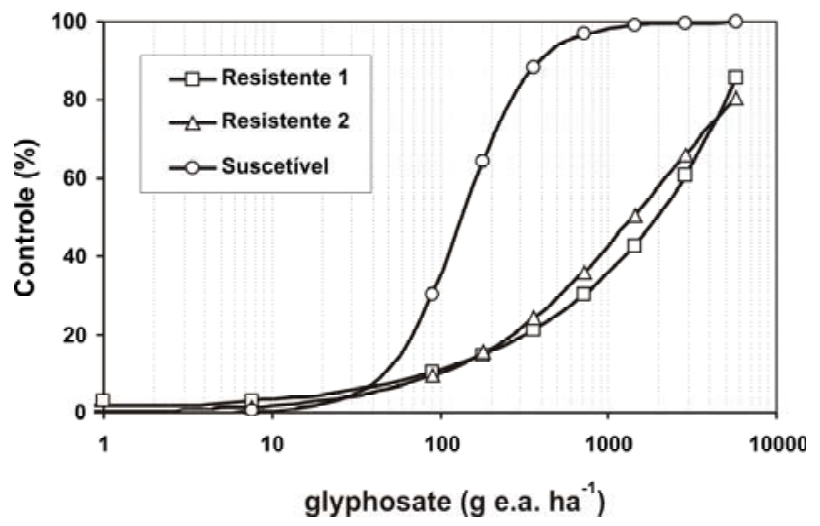

Figura 3 - Controle percentual de três populações de Conyza bonariensis, duas supostamente resistentes e uma suscetível, quando submetidas a diferentes doses do herbicida glyphosate, avaliado aos 28 DAA. DMS ${ }_{\text {populacão }}=9,00$. Piracicaba, 2006.

Novamente os dados de massa seca residual estão em concordância com os valores de controle obtidos, em que foi necessária a aplicação de doses mais elevadas sobre as populações de $C$. bonariensis coletadas em pomares de citros do Estado de São Paulo, quando comparadas com a população considerada suscetível, coletada em área sem histórico de aplicação de glyphosate (Figura 4). Ainda, também para esta espécie de buva, todos os tratamentos alternativos alcançaram $100 \%$ de 


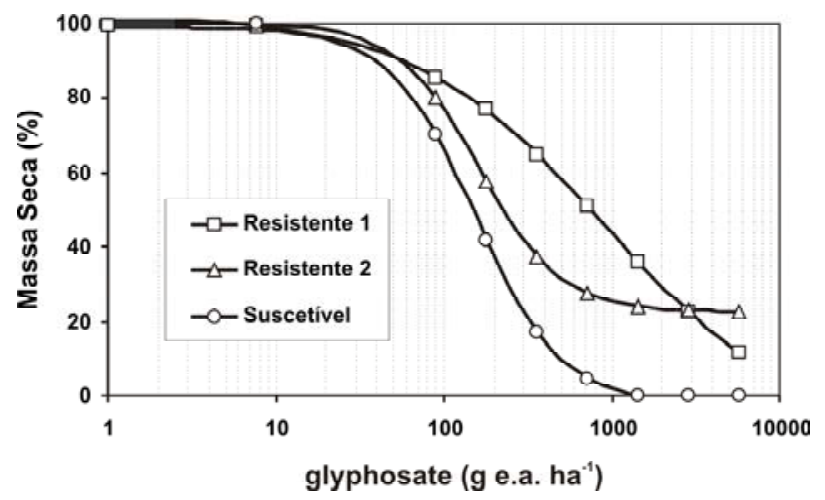

Figura 4 -Massa seca residual(\%) de três populações de Conyza bonariensis, duas supostamente resistentes e uma suscetível, quando submetidas a diferentes doses do herbicida glyphosate, avaliada aos 28 DAA. DMS população $=30,2$. Piracicaba, 2006

controle de todas as populações, na avaliação realizada aos 28 DAA, sendo considerados opções viáveis para o manejo de populações resistentes (dados não apresentados).

\section{Niveis de resistência}

Na Tabela 3 são apresentados os parâmetros dos modelos logísticos ajustados para os controles obtidos com a aplicação das diferentes doses de glyphosate sobre todas as populações de C. canadensis e C. bonariensis. Com esses dados, puderam ser calculados matematicamente os valores de $\mathrm{C}_{50}$ ou $\mathrm{GR}_{50}$ (Carvalho et al., 2005), que caracterizaram os niveis de suscetibilidade das populações de plantas daninhas ao herbicida.

Para a espécie $C$. canadensis, observa-se que as populações coletadas nos pomares de citros (R1 e R2), mantidas sob manejo com o herbicida glyphosate, apresentaram fatores de resistência da ordem de 10,79 e 7,09, para a variável controle percentual; e 6,15 e 6,65, para a variável massa seca residual, respectivamente (Tabela 3). VanGessel (2001) e Koger et al. (2004) encontraram resultados semelhantes nos Estados Unidos, onde observaram fator de resistência de aproximadamente 10 vezes para populações de $C$. canadensis.

Observando os resultados obtidos para C. bonariensis, pôde-se verificar que o fator de resistência caracterizado para as populações supostamente resistentes dessa planta daninha (R1 e R2) foi de 14,75 e 10,40, para a variável controle percentual, e 5,02 e 1,52, para a variável massa seca residual, respectivamente (Tabela 3). De forma geral, todos os fatores de resistência obtidos caracterizaram populações selecionadas pela aplicação rotineira do herbicida glyphosate e, portanto, menos sensiveis

Tabela 3 - Parâmetros dos modelos logísticos ${ }^{1^{\prime}}$, doses que obtiveram $50 \%$ de controle $\left(\mathrm{C}_{50}\right)$ ou de redução de crescimento $\left(\mathrm{GR}_{50}\right)$ e fator de resistência R/S $(\mathrm{F})$, obtidos para todas as populações de Conyza canadensis e C. bonariensis, quando submetidas a diferentes doses do herbicida glyphosate. Piracicaba, 2006

\begin{tabular}{|c|c|c|c|c|c|c|c|}
\hline \multirow{2}{*}{ Variável } & \multirow{2}{*}{ População } & \multicolumn{4}{|c|}{ Parâmetro } & \multirow{2}{*}{$\mathrm{C}_{50}$ ou $\mathrm{GR}_{50}$} & \multirow{2}{*}{$\mathrm{F}$} \\
\hline & & $\mathrm{a}$ & $\mathrm{b}$ & $\mathrm{c}$ & $\mathrm{d}$ & & \\
\hline \multicolumn{8}{|c|}{ Conyza canadensis } \\
\hline \multirow{3}{*}{ Controle $(\%)$} & Resistente 1 & 94,26 & $1.376,04$ & $-0,92$ & - & $1.570,51$ & 10,79 \\
\hline & Resistente 2 & 90,80 & 931,33 & $-1,98$ & - & $1.032,01$ & 7,09 \\
\hline & Suscetível & 102,11 & 148,87 & $-1,83$ & - & $1.45,55$ & - \\
\hline \multirow{3}{*}{ Massa Seca (\%) } & Resistente 1 & $-11,78$ & 112,19 & 639,59 & 0,53 & 435,85 & 6,15 \\
\hline & Resistente 2 & 13,82 & 87,56 & 306,08 & 0,81 & 471,47 & 6,65 \\
\hline & Suscetível & 0,65 & 99,35 & 70,40 & 2,07 & 70,85 & - \\
\hline \multicolumn{8}{|c|}{ Conyza bonariensis } \\
\hline \multirow{3}{*}{ Controle $(\%)$} & Resistente 1 & $1.741,27$ & $1.705 .131,67$ & $-0,52$ & - & $1.992,35$ & 14,75 \\
\hline & Resistente 2 & 116,11 & $2.011,86$ & $-0,78$ & - & $1.404,50$ & 10,40 \\
\hline & Suscetível & 99,83 & 134,82 & $-2,07$ & - & $1.35,04$ & - \\
\hline \multirow{3}{*}{ Massa Seca (\%) } & Resistente 1 & $-9,51$ & 111,69 & 886,77 & 0,77 & 746,60 & 5,02 \\
\hline & Resistente 2 & 22,46 & 77,62 & 162,38 & 1,79 & 226,80 & 1,52 \\
\hline & Suscetível & $-2,29$ & 101,30 & 154,55 & 1,70 & 148,77 & - \\
\hline
\end{tabular}

${ }^{1 /}$ Variável controle - modelo: $\mathrm{y}=\left(\mathrm{a} /\left(1+(\mathrm{x} / \mathrm{b})^{\mathrm{c}}\right)\right)$; Variável massa seca - modelo: $\mathrm{y}=\mathrm{a}+\left(\mathrm{b} /\left(1+(\mathrm{x} / \mathrm{c})^{\mathrm{d}}\right)\right)$. 
à ação deste, exceto a população resistente 2 , para a variável massa seca residual, em que o nivel de resistência foi menos expressivo $(1,52)$.

Embora seja comercializado desde a década de 1970, o primeiro caso de planta daninha resistente ao glyphosate foi relatado somente em 1996 (Pratley et al., 1996). Isso pode ser explicado, principalmente, pelas características bioquímicas da molécula quando presente nas plantas ou no solo, como: ausência de atividade residual no solo, presença de múltiplos mecanismos fisiológicos correlacionados com o mecanismo de ação, baixa adaptabilidade ecológica dos indivíduos sobreviventes, baixa freqüência inicial de indivíduos resistentes, ausência de outros herbicidas com o mesmo mecanismo de ação e metabolis mo limitado pelas plantas (Bradshaw et al., 1997).

A resistência de populações de plantas daninhas a herbicidas aplicados em pós-emergência pode estar relacionada com a afinidade enzimática das moléculas; com a absorção, translocação ou exclusão diferencial dos herbicidas; ou mesmo com rotas de detoxificação metabólica. Neste caso, ainda não se sabe ao certo qual é o mecanismo envolvido na resistência. Segundo Feng et al. (2004), a translocação reduzida do glyphosate para os pontos de crescimento da planta e para as raízes, juntamente com a possivel compartimentalização do produto dentro da planta, são mecanismos responsáveis pela resistência. Contudo, maiores estudos devem ser realizados para esclarecer quais fatores têm participação na resposta diferencial de controle das populações brasileiras de Conyza ao herbicida glyphosate.

Assim, a partir dos resultados obtidos, comprovou-se a existência de populações brasileiras de Conyza canadensis e C. bonariensis com ocorrência de biótipos resistentes ao herbicida glyphosate, com diferentes niveis de resistência. Todos os tratamentos herbicidas alternativos controlaram de forma eficiente as três populações de cada espécie.

\section{LITERATURA CITADA}

BHOWMIK, P. C.; BEKECH, M. M. Horseweed (Conyza canadensis) seed production, emergence and distribuition in no-tillage and conventional-tillage corn (Zea mays).

Agronomy, v. 1, p. 67-71, 1993.
BLACKSHAW, R. E.; HARKER, K. N. Selective weed control with glyphosate in glyphosate-resistant spring wheat (Triticum aestivum). Weed Technol., v. 16, p. 885-892, 2002.

BRADSHAW, L. D. et al. Perspectives on glyphosate resistance. Weed Technol., v. 11, p. 189-198, 1997.

CARVALHO, S. J. P. et al. Curvas de dose-resposta para avaliação do controle de fluxos de emergência de plantas daninhas pelo herbicida imazapic. Planta Daninha, v. 23, n. 3, p. 535-542, 2005.

CHRISTOFFOLETI, P. J. et al. Alternative herbicides to manage Italian ryegrass (Lolium multiflorum Lam.) resistant to glyphosate at different phonological stages. J. Environ. Sci. Health, v. B40, p. 59-67, 2005.

CHRISTOFFOLETI, P. J.; LÓPEZ-OVEJERO, R. F. Definições e situação da resistência de plantas daninhas aos herbicidas no Brasil e no mundo. In: CHRISTOFFOLETI, P. J. (Coord.). Aspectos de resistência de plantas daninhas a herbicidas. 2.ed. Campinas: Associação Brasileira de Ação a Resistência de Plantas aos Herbicidas (HRAC-BR), 2004. p. 3-22.

CHRISTOFFOLETI, P. J. Curvas de dose-resposta de biótipos resistente e suscetível de Bidens pilosa L. aos herbicidas inibidores da ALS. Sci. Agric., v. 59, n. 3, p. 513-519, 2002.

CHRISTOFFOLETI, P. J.; VICTORIA FILHO, R.; SILVA, C. B. Resistência de plantas daninhas aos herbicidas. Planta Daninha, v. 12, n. 1, p. 13-20, 1994.

FAIRCLOTH, W. H. et al. Weed management programs for glyphosate-tolerant cotton (Gossipium hirsutum). Weed Technol., v. 15, p. 544-551, 2001.

FENG, P. C. C. et al. Investigations into glyphosate resistant horseweed (Conyza canadensis): retention, uptake, translocation and metabolism. Weed Sci., v. 52, p. 498-505, 2004.

GEIGER, D. R.; FUCHS, M. A. Inhibitors of aromatic amino acid biosynthesis (glyphosate). In: BÖGER, P.; WAKABAYASHI, K.; HIRAI, K. (Ed.). Herbicide classes in development. Berlin: Springer-Verlag, 2002. p. 59-85.

HALL, L. M.; STROME, K. M.; HORSMAN, G. P. Resistance to acetolactate synthase inhibitors and quinclorac in a biotype of false clover (Gallium spurium). Weed Sci., v. 46, p. 390-396, 1998.

HEAP, I. The international survey of herbicide resistant weeds. Disponible: $<$ www.weedscience.com>. Acessible: 10 nov. 2004. 
KOGER, C. H. et al. Glyphosate resistant horseweed (Conyza canadensis) in Mississipi. Weed Technol., v. 18, p. 820-825, 2004.

KOGER, C. H.; REDDY, K. N. Role of absorption and translocation in the mechanism of glyphosate resistance in horseweed (Conyza canadensis). Weed Sci., v. 53, p. 84-89, 2005.

LEE, L. J.; NGIM, J. A first report of glyphosate-resistant goosegrass (Eleusine indica) in Malaysia. Pest Manag. Sci., v. 56, p. 336-339, 2000.

MAIN, C. L. et al. Glyphosate-resistant Italian ryegrass x 779 response of selected horseweed (Conyza canadensis (L.) Cronq.) populations to glyphosate. J. Agric. Food Chem., v. 52, p. 879-883, 2004.

MUELLER, T. C. et al. Shikimate accumulation in both glyphosate-sensitive and glyphosate-resistant horseweed (Conyza canadensis L. Cronq). J. Agric. Food Chem., v. 51, p. 680-684, 2003.

PEREZ, A.; KOGAN, M. Glyphosate-resistant Lolium multiflorum in Chilean orchards. Weed Res., v. 43, p. 12-19, 2003.

PEREZ-JONES, A. et al. Glyphosate- resistant Lolium multiflorum in Oregon. Proc. West. Soc. Weed Sci., p. 57-27, 2004.

POWLES, S. B. et al. Evolved resistance to glyphosate in rigid ryegrass (Lolium rigidum) in Australia. Weed Sci., v. 46, p. 604-607, 1998.

PRATLEY, J. et al. Glyphosate resistance in annual ryegrass. In: ANNUAL CONFERENCE OF THE GRASSLAND SOCIETY OF NEW SOUTH WALES, 11., Wagga Wagga, 1996. Proceedings... Wagga Wagga: The Grassland Society of New South Wales, 1996. p. 126.
PRATLEY, J. et al. Resistance to glyphosate in Lolium rigidum. I. Bioevaluation. Weed Sci., v. 47, p. 405-411, 1999.

SEEFELDT, S. S.; JENSEN, S. E.; FUERST, E. P. Loglogistic analysis of herbicide dose-response relationship. Weed Technol., v. 9, p. 218-227, 1995.

SELLERS, B. A.; POLLARD, J. M.; SMEDA, R. J. Two common ragweed (Ambrosia artemisiifolia) biotypes differ in biology and response to glyphosate. Proc. Weed Sci. Soc., v. 45 , p. 156, 2005.

SIMARMATA, M.; KAUFMANN, J. E.; PENNER, D. Potential basis of glyphosate resistance in California rigid ryegrass (Lolium rigidum). Weed Sci., v. 51, p. 678-682, 2003.

STREIBIG, J. C. Herbicide bioassay. Weed Res., v. 28, n. 6, p. $479-484,1988$.

TRAN, M. et al. Characterization of glyphosate resistant Eleusine indica biotypes from Malaysia. In: ASIANPACIFIC WEED SCIENCE SOCIETY SOCIETY CONFERENCE, 17., 1999, Bangkok. Proceedings... Bangkok: Asian-Pacific Weed Science Society, 1999. p. 527-536.

URBANO, J. M. et al. Glyphosate-resistant hairy fleabane (Conyza bonariensis) in Spain. Proc. Weed Sci. Soc., v. 45, p. 394, 2005.

VANGESSEL, M. J. Glyphosate resistant horseweed from Delaware. Weed Sci., v. 49, p. 703-705, 2001.

WEAVER, S. E. The biology of Canadian weeds, Conyza canadensis. Can. J. Plant Sci., v. 81, p. 867-875, 2001. 